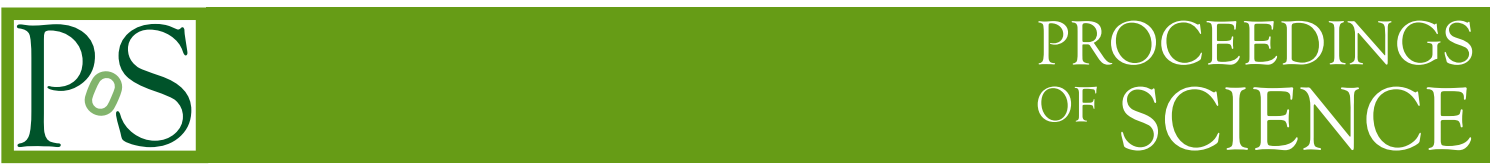

\title{
W boson measurements with the CMS experiment
}

\author{
Riccardo Salvatico ${ }^{a, b, 1, *}$ \\ ${ }^{a}$ Università degli Studi di Torino, \\ Via Giuria 1, Torino, Italy \\ ${ }^{b}$ INFN, \\ Via Giuria 1, Torino, Italy \\ E-mail: riccardo.salvatico@cern.ch
}

The $\mathrm{W}$ boson, one of the two mediators of the weak interaction, has been broadly studied at both $\mathrm{e}^{+} \mathrm{e}^{-}$and hadron colliders since its discovery. Despite the increasing accuracy in the measurement of its properties, it still remains relatively poorly known if compared to the other mediator, the $\mathrm{Z}$ boson, due to the bigger experimental challenges in the reconstruction of the $\mathrm{W}$ boson decays. The CMS experiment at the CERN LHC covers a vast program in the electroweak physics sector, with numerous analyses aiming at measuring the $\mathrm{W}$ boson properties with high precision. Some of the latest results from the CMS Collaboration are presented in this report. These include measurements of the multi-differential $\mathrm{W}$ boson production cross sections, polarization and charge asymmetry, the first search for the rare decay of the $\mathrm{W}$ boson into a pion and a photon at the LHC, and the first evidence for WW production via double-parton scattering. Such measurements exploit, either partially or fully, the integrated luminosity collected by CMS during the LHC Run 2 (2016-2018), and allow us to further increase our knowledge of this fundamental piece of the standard model of particle physics.

40th International Conference on High Energy Physics - ICHEP2020

July 28 - August 6, 2020

Prague, Czech Republic (virtual meeting)

${ }^{1}$ For the CMS Collaboration

${ }^{*}$ Speaker 


\section{W boson rapidity, helicity, and differential cross sections}

Several properties of the W boson are measured in Ref. [1] using data collected with the CMS detector [2] in proton-proton (pp) collisions at $\sqrt{s}=13 \mathrm{TeV}$, corresponding to an integrated luminosity of about $36 \mathrm{fb}^{-1}$. These include the $\mathrm{W}$ boson rapidity and helicity, differential and double-differential production cross sections, and charge asymmetry. The polarization of the $\mathrm{W}$ boson is a cornerstone in the determination of its production mechanism, and a precise knowledge of the $\mathrm{W}$ boson rapidity $\left(y_{\mathrm{W}}\right)$, helicity, and charge asymmetry may help to constrain the uncertainties on the parton distribution functions (PDFs) of the proton, which strongly affect the precision of the measurements of the $\mathrm{W}$ boson mass at hadron collider experiments.

The $\mathrm{W}$ boson candidates are reconstructed through their leptonic decays into a muon or an electron plus a neutrino. Two-dimensional (2D) templates in the charged-lepton pseudorapidity $\left(\eta^{\ell}\right)$ and transverse momentum $\left(p_{\mathrm{T}}^{\ell}\right)$ are built for each $\mathrm{W}$ boson polarization state, and then fitted to the observed 2D distributions in data. The rapidity-dependent cross sections $\mathrm{d} \sigma / \mathrm{d} y_{\mathrm{W}}$ are measured separately for the two charge signs of the $\mathrm{W}$ boson and for its left and right helicity states, whereas the longitudinal polarization component is fixed to the prediction of the MAdGRAPH5_amC@NLO Monte Carlo (MC) generator [3] with a 30\% normalization uncertainty. The electron and muon channels are combined, and a ratio is performed to the fitted total inclusive $\mathrm{W}$ production cross section, so that a partial cancellation of the uncertainties correlated among the rapidity bins is achieved. The $\mathrm{W}$ boson charge asymmetry for each polarization (pol) state is derived from the $\left|y_{\mathrm{W}}\right|$-dependent cross section, following

$$
\mathcal{A}^{\mathrm{pol}}\left(\left|y_{\mathrm{W}}\right|\right)=\frac{\mathrm{d} \sigma^{\mathrm{pol}} / \mathrm{d}\left|y_{\mathrm{W}}\right|\left(\mathrm{W}^{+} \rightarrow \ell^{+} v\right)-\mathrm{d} \sigma^{\mathrm{pol}} / \mathrm{d}\left|y_{\mathrm{W}}\right|\left(\mathrm{W}^{-} \rightarrow \ell^{-} \bar{v}\right)}{\mathrm{d} \sigma^{\mathrm{pol}} / \mathrm{d}\left|y_{\mathrm{W}}\right|\left(\mathrm{W}^{+} \rightarrow \ell^{+} v\right)+\mathrm{d} \sigma^{\mathrm{pol}} / \mathrm{d}\left|y_{\mathrm{W}}\right|\left(\mathrm{W}^{-} \rightarrow \ell^{-} \bar{v}\right)} .
$$
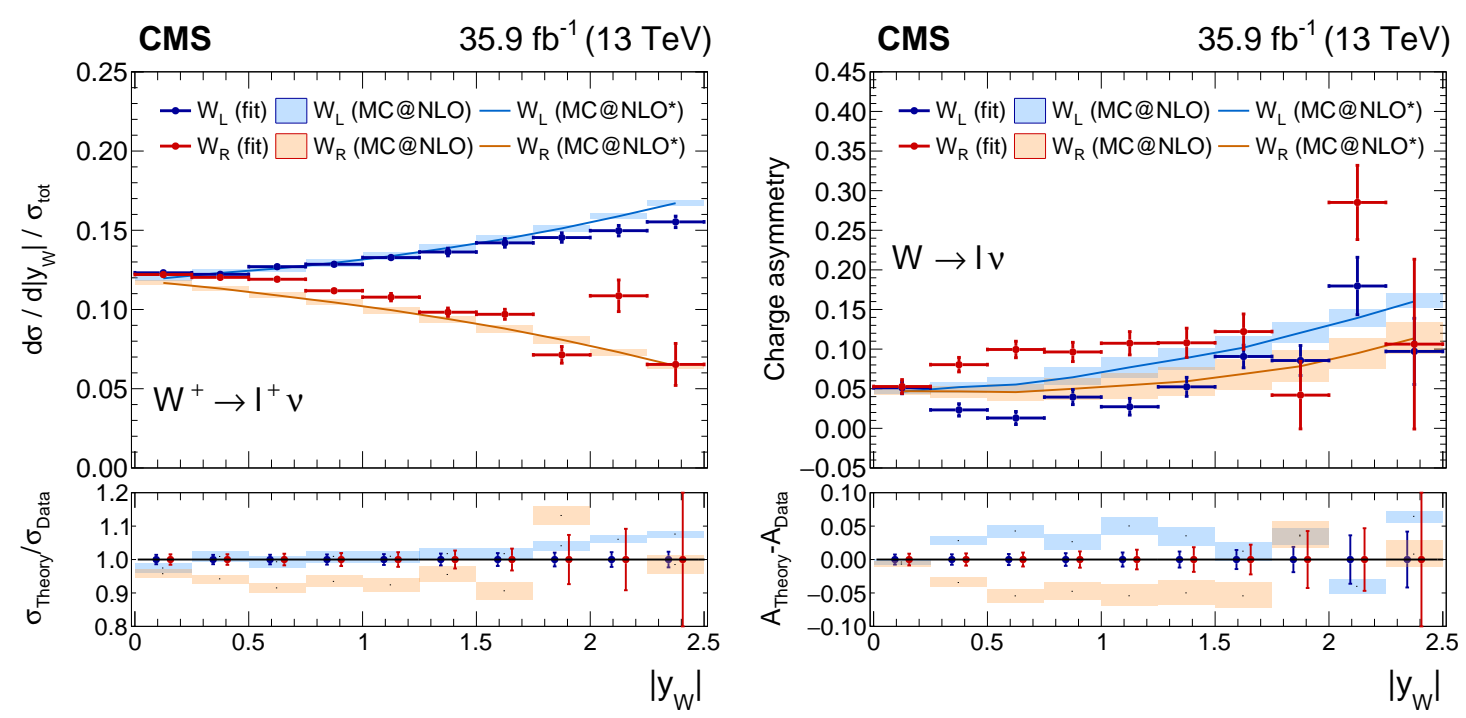

Figure 1: Differential $\mathrm{W}^{+}$boson production cross section as a function of its rapidity and normalized to the total production cross section (left); W boson charge asymmetry as a function of its rapidity (right) [1]. The results are split in the left and right polarization states of the $\mathrm{W}$ boson, with the blue and orange bands representing the theoretical prediction obtained using the MADGRAPH5_aMC@NLO generator. 


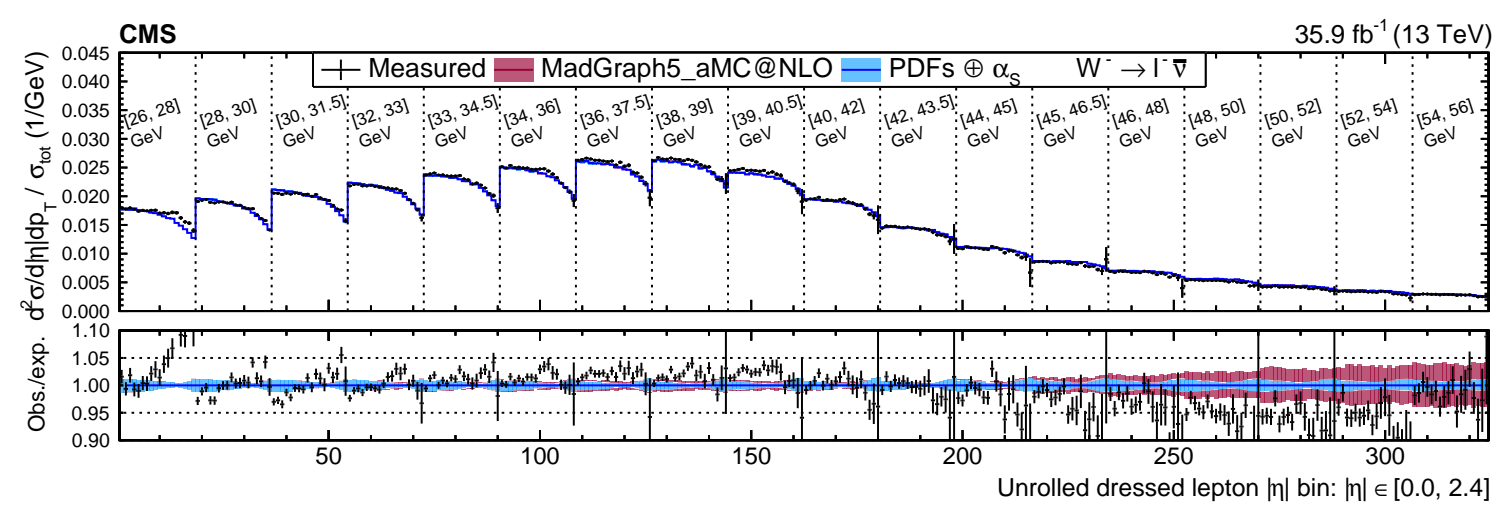

Figure 2: Normalized double-differential cross section as a function of $p_{\mathrm{T}}^{\ell}$ and $\left|\eta^{\ell}\right|$, unrolled in a onedimensional histogram along $\left|\eta^{\ell}\right|$ for the negative charge [1]. The lower panel shows the ratio of the observed and expected cross sections. The red bands represent the prediction from MADGRAPH5_amC@NLO.

For the first time, this quantity is unfolded in rapidity bins and split in the right and left helicity states. The rapidity-dependent $\mathrm{W}^{+}$production cross section and the measured $\mathrm{W}$ boson charge asymmetry are shown in Fig. 1.

Double-differential production cross sections are measured for each charge of the $\mathrm{W}$ boson, in very fine bins of $\left|\eta^{\ell}\right|$ and $p_{\mathrm{T}}^{\ell}$. The underlying templates are unfolded to the generated lepton kinematic properties using a procedure in which electroweak radiation is added back to the chargedlepton momentum within a cone of $\Delta R \equiv \sqrt{(\Delta \eta)^{2}+(\Delta \phi)^{2}}<0.1$. Such measurements represent a rigorous test of the most recent predictions obtained from theoretical calculations at next-to-next-toleading order accuracy in perturbative quantum chromodynamics (QCD), and a direct comparison with the prediction of MAdGraph5_amc@NLo (Fig. 2) shows an agreement at the level of 1\% in the central part of the lepton acceptance $\left(\left|\eta^{\ell}\right|<1\right)$. Similarly to the $\left|y_{\mathrm{W}}\right|$-dependent case, the double-differential $\mathrm{W}$ boson charge asymmetry is computed as well.

Finally, a test is performed to evaluate the statistical power of the fit in constraining the PDF uncertainties. For this purpose, the cross sections are fixed to their expected values, and several parameters corresponding to NNPDF3.0 [4] replicas are floated. A mean post-fit constraint of $\approx 90 \%$ of the pre-fit values is observed. This is a proof of principle that the PDF uncertainties can be reduced to target a measurement of the $\mathrm{W}$ mass with a precision at the $\mathrm{MeV}$ level.

\section{The rare decay $\mathrm{W} \rightarrow \pi \gamma$}

The rare decay $\mathrm{W} \rightarrow \pi \gamma$, like any other exclusive hadronic decay of the $\mathrm{W}$ boson predicted by the standard model (SM) of particle physics, has never been observed. The theoretical predictions for its branching fraction $\left(\mathcal{B}\right.$ ) have large uncertainties, and set it in the range $10^{-9}-10^{-7}$ [5]. Nevertheless, this rare decay represents an interesting probe of the strong interaction at the boundary between the perturbative and nonperturbative domains of QCD, and its observation would offer insights into factorization and meson form factors at large energy scales. Furthermore, at future colliders it could be used for a measurement of the mass of the $\mathrm{W}$ boson that is based solely on visible single-particle decay products. 


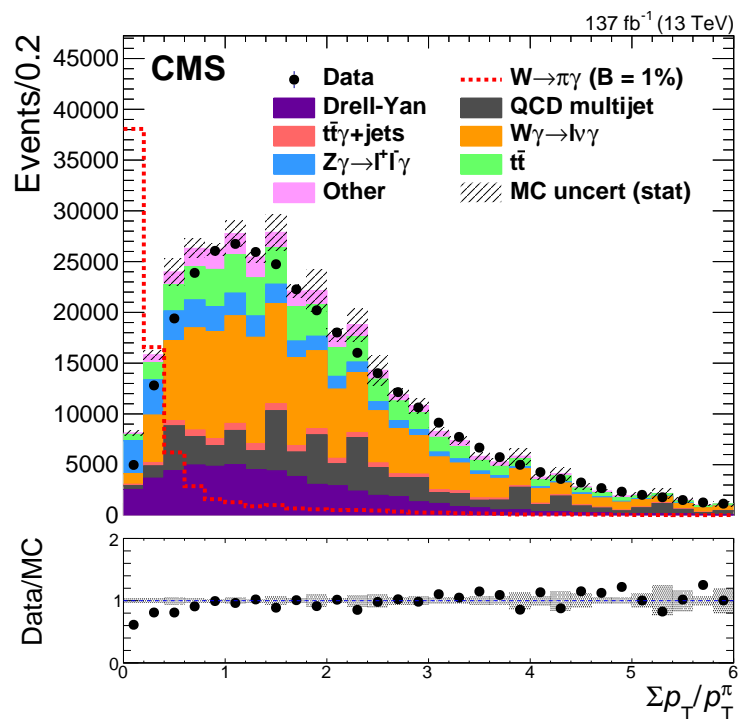

Figure 3: Event distribution as a function of the pion-isolation variable $\Sigma p_{\mathrm{T}} / p_{\mathrm{T}}^{\pi}$ [6]. The simulated MC distribution for the signal is given by the dashed red line and corresponds to a $1 \%$ branching fraction for the $\mathrm{W} \rightarrow \pi \gamma$ decay. The statistical uncertainties in the data are small and thus not visible.

Given the high energy thresholds for single photon triggers, which make an inclusive search unsuitable at CMS, a novel search strategy is adopted in Ref. [6], focusing on W boson production from top quark-antiquark pair (tît) events, using an integrated luminosity of $137 \mathrm{fb}^{-1}$. The muon or electron emerging from the leptonic decay of one of the two $\mathrm{W}$ bosons from the $\mathrm{t} \overline{\mathrm{t}}$ pair is used to select events at the trigger level, and the presence of $\mathrm{b}$ quark jets is used to reduce the background from the hadronization of light-flavor quarks and gluons. Finally, the other W boson in the event is employed to search for the rare decay topology, characterized by an isolated photon of high $p_{\mathrm{T}}$, and a high- $p_{\mathrm{T}}$ isolated track.

The analysis exploits the minimal amount of jet activity expected in the vicinity of the pion, which is produced via electroweak processes, to reject background pions. Thus, a pion-isolation variable is implemented, based on the sum of the $p_{\mathrm{T}}$ of all the particles contained in a cone with radius $0.02<\Delta R<0.5$ around the pion, divided by the pion $p_{\mathrm{T}}\left(\Sigma p_{\mathrm{T}} / p_{\mathrm{T}}^{\pi}\right)$. Figure 3 shows the distribution of the pion-isolation variable for signal events, which appear to be highly isolated, while background events indicate a clear presence of jet activity.

Events are classified in a muon and an electron channel, depending on the flavor of the triggering lepton. A set of variables with good signal/background discriminating power, including the lepton, pion and photon momenta, the number of $b$ jets, and the pion isolation, is then used to train one boosted decision tree (BDT) per lepton channel. Upon the output of these BDTs, a signal- and a background-enriched regions of the pion-photon invariant mass $\left(m_{\pi \gamma}\right)$ are defined. The latter is used to extract the background lineshape directly from collision data, whereas the signal functional form is described using simulated events. The distribution of $m_{\pi \gamma}$ is fitted in the signal region (see Fig. 4) to measure $\mathcal{B}(\mathrm{W} \rightarrow \pi \gamma)$. No significant excess is observed above the expected background. An upper limit at $95 \%$ confidence level $\mathcal{B}(\mathrm{W} \rightarrow \pi \gamma)<1.50 \times 10^{-5}$ is set. This result demonstrates the feasibility of a search for such rare decays of the $\mathrm{W}$ bosons at the LHC and defines a suitable 


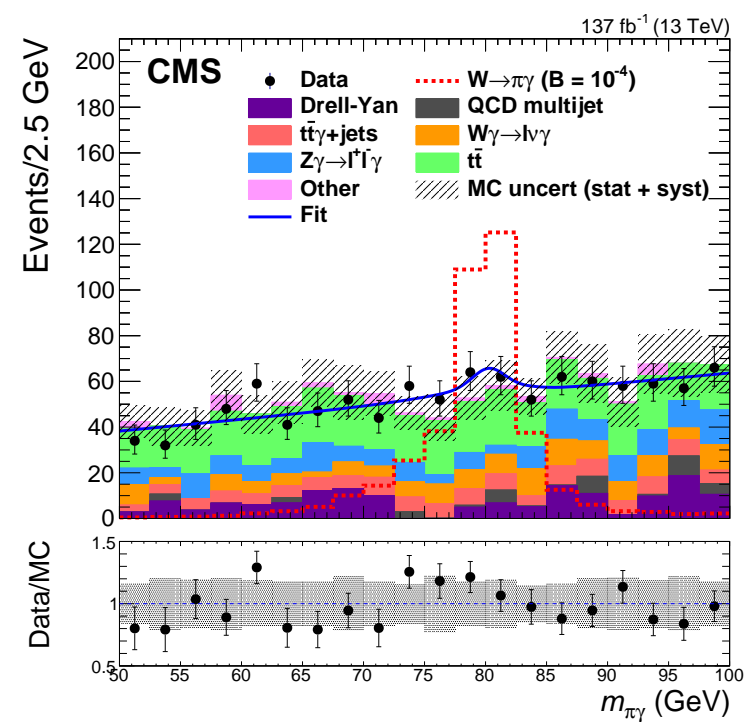

Figure 4: Event distribution as a function of $m_{\pi \gamma}$ for the combination of the lepton channels [6]. The simulated MC distribution for the signal is given by the dashed red line and corresponds to a $\mathcal{B}(\mathrm{W} \rightarrow \pi \gamma)=10^{-4}$. The blue line represents the best fit to the data.

search strategy.

\section{WW production from double-parton scattering}

A double-parton scattering (DPS) occurs when two hard parton-parton interactions take place within a single pp collision. A particularly promising way to search for DPS events at CMS is when both hard scatterings lead to the production of same-sign W bosons, observed via their leptonic decay. In such a scenario, the single hard scattering (SHS) production of same-sign W bosons is suppressed at matrix element level, and the absence of jets in DPS $\mathrm{W}^{ \pm} \mathrm{W}^{ \pm}$production at leading order in perturbation theory can be used to suppress other SHS backgrounds; furthermore, two same-sign leptons provide a somewhat clean final state in the detector and can be reconstructed with high accuracy.

The analysis in Ref. [7] exploits an integrated luminosity of about $77 \mathrm{fb}^{-1}$ and uses a combination of dilepton and single-lepton triggers to select events of interest. The dilepton final states considered are $\mu \mu$ and e $\mu$, whereas the ee final state is not examined because of the higher level of background in this channel. The event selection proceeds through two BDTs, trained to separate the signal from the dominant background contributions: the $\mathrm{WZ}$ associated production, and those processes that generate nonprompt leptons (W+ jets, QCD multijet etc.). The two multivariate classifiers are then combined and mapped into the one-dimensional classifier that exhibits the largest expected significance. A maximum likelihood fit is performed simultaneously on the four independent distributions of this classifier, given by the two lepton flavor and charge configurations, and the DPS $\mathrm{W}^{ \pm} \mathrm{W}^{ \pm}$cross section is extracted. The values obtained are then extrapolated to the inclusive WW phase space, and compared with the predictions obtained with PYTHIA 8 [8] and with the factorization approach, used by several MC generators (see Fig. 5). 


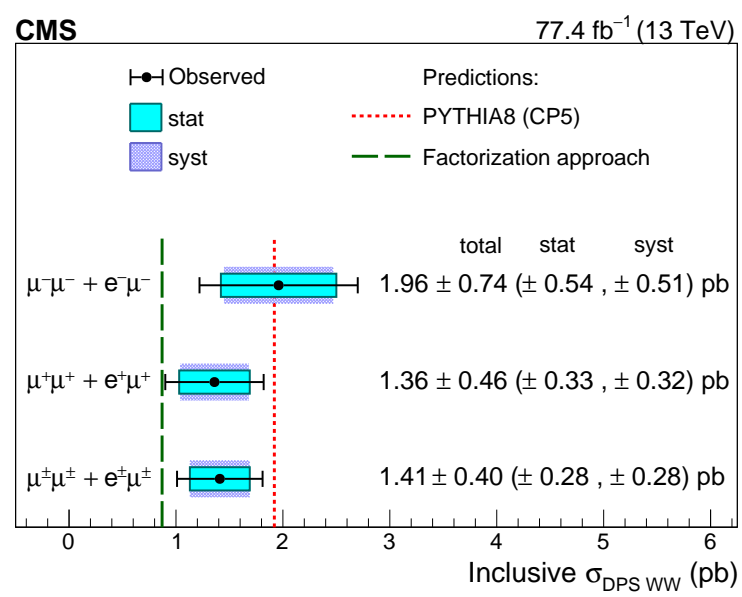

Figure 5: Observed cross section values for inclusive DPS WW production from the two lepton charge configurations and their combination [7].

With an observed significance of 3.9 standard deviations, these results represent the first evidence for WW production from DPS. These measurements will help to validate the factorization approach and will permit a more precise determination of the contribution of WW production from DPS in searches for new physics.

\section{References}

[1] CMS Collaboration, Measurements of the $W$ boson rapidity, helicity, double-differential cross sections, and charge asymmetry in pp collisions at $\sqrt{s}=13 \mathrm{TeV}$, CERN-EP-2020-116 [2008.04174].

[2] CMS Collaboration, The CMS experiment at the CERN LHC, JINST 3 (2008) S08004.

[3] J. Alwall et al., The automated computation of tree-level and next-to-leading order differential cross sections, and their matching to parton shower simulations, JHEP 07 (2014) 079 [1405.0301].

[4] NNPDF Collaboration, Parton distributions for the LHC Run II, JHEP 04 (2015) 040 [1410.8849].

[5] M. Mangano and T. Melia, Rare exclusive hadronic $W$ decays in a t $\bar{t}$ environment, Eur. Phys. J. C 75 (2015) 258 [1410.7475].

[6] CMS Collaboration, Search for the rare decay of the $W$ boson into a pion and a photon in proton-proton collisions at $\sqrt{s}=13 \mathrm{TeV}$, CERN-EP-2020-197 [2011.06028].

[7] CMS Collaboration, Evidence for WW production from double-parton interactions in protonproton collisions at $\sqrt{s}=13 \mathrm{TeV}$, Eur. Phys. J. C 80 (2020) 41 [1909. 06265].

[8] T. Sjöstrand et al., An introduction to PYTHIA 8.2, Comput. Phys. Commun. 191 (2015) 159 [1410.3012]. 